\title{
Evaluación y calidad educativa: Avances, limitaciones y retos actuales
}

\author{
Assessment and Educational Quality: Advances, Limitations and Current Challenges
}

\section{Avaliação e qualidade educacional: avanços, limitações e desafios atuais}

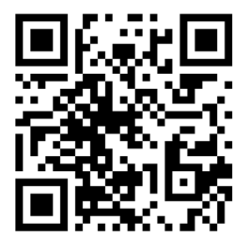

Dania Liz Mejía-Rodríguez

Universidad Simón Bolívar

Barranquilla, Colombia

dmejia22@unisimonbolivar.edu.co

https://orcid.org/0000-0003-3302-0254

Ever Javier Mejía-Leguía

Universidad de la Costa

Barranquilla, Colombia

emejia13@cuc.edu.co

https://orcid.org/0000-0002-4627-6980

Recibido • Received • Recebido: 10 / 11 / 2019

Corregido • Revised • Revisado: 17 / 07 / 2021

Aceptado • Accepted • Aprovado: 12 / 08 / 2021

\begin{abstract}
Resumen: El presente ensayo diserta sobre el panorama actual de la evaluación y la calidad educativa en términos de sus avances, limitaciones y retos. Para ello se alude al papel de la evaluación en el mejoramiento de los procesos educativos, y su repercusión en la consolidación de la calidad educativa en América Latina. A partir de ello se identifica que la evaluación en el ámbito educativo se ha visto fortalecida, tanto en su concepción como en sus implicaciones, frente a la búsqueda de mejoramiento continuo de la educación, así como de las acciones que contribuyan a procesos de cambio desde el espacio educativo. Asimismo, la calidad educativa empieza a asumirse como un constructo más enfocado a la transformación reflexiva de la praxis mediante acciones contextualizadas. Sin embargo, aún persisten limitaciones relacionadas con la ambigüedad en los propósitos, métodos y alcances de la evaluación, lo que repercute en el potencial de su aprovechamiento para fortalecer la calidad educativa. De allí que los retos apunten a la articulación intencionada, rigurosa y pertinente de la evaluación en los procesos de fortalecimiento educativo.
\end{abstract}

Palabras claves: Calidad de la educación; evaluación; América Latina; educación. 
http://doi.org/10.15359/ree.25-3.38

http://www.una.ac.cr/educare

educare@una.ac.cr

\begin{abstract}
This essay discusses current advances, challenges, and limitations about educational assessment and quality. For this purpose, the assessment role in educational processes improvement and their effects on educational quality was identified. Main findings show that conceptions about assessment and their applications in education have advanced; they have contributed to the continuous progress of education practices. Likewise, educational quality has been related to reflexive changes through contextual practices. However, at present, some limitations on purposes, methods, scopes, and potential uses of assessment for educational quality persist. Hence, challenges relate to the intended, rigorous, and pertinent relationship between assessment and educational improvement.
\end{abstract}

Keywords: Educational quality; assessment; Latin America; education.

Resumo: O presente ensaio disserta sobre o panorama atual da avaliação e a qualidade educativa em termos de seus avanços, limitações e desafios. Para tal, faz-se referência ao papel da avaliação na melhoria dos processos educativos e ao seu impacto na consolidação da qualidade educativa. A partir disto, identifica-se que a avaliação no âmbito educativo foi fortalecida tanto na sua concepção como nas suas implicações frente à busca de melhoria contínua da educação, bem como das ações que contribuam para a transformação social a partir do âmbito educativo. Da mesma forma, a qualidade educativa começa a assumir-se como um construto mais focalizado na transformação reflexiva da práxis mediante ações contextualizadas. No entanto, ainda persistem limitações em torno dos propósitos, métodos, alcances e usos potenciais da avaliação para a qualidade educacional. A partir disso, os desafios direcionam a articulação deliberada, rigorosa e pertinente de avaliação nos processos de fortalecimento educacional.

Palavras chaves: Qualidade educativa; avaliação; América Latina; educação.

\title{
Introducción
}

Ante el creciente interés por consolidar procesos evaluativos que permean la educación desde sus distintos escenarios y funciones, se han generado acciones que, si bien aportan a la identificación de factores asociados al quehacer en este ámbito, también se han formado interpretaciones desacertadas en torno a cómo se asume la evaluación y frente a las conclusiones o decisiones que se toman a partir de los resultados obtenidos en ellas. Frente a este punto cabe anotar que los sistemas de evaluación se estructuran desde una serie de intereses entre los que se destacan 1) la formulación de políticas educativas fundamentadas en datos objetivos; 2) fortalecer la gestión de los sistemas educativos actuales; 3) promover la autorreflexión y el aprendizaje continuo en las instituciones educativas y organizaciones relacionadas con el propósito de formar seres humanos para una ciudadanía y sujetos de transformación social (Ravela et al., 2008).

Coherentes con estos intereses, y desde la perspectiva de la calidad educativa, la evaluación se constituye como un campo disciplinar desde el que se estructura la construcción de conocimientos rigurosos tendientes a la identificación y el monitoreo de avances, retrocesos, falencias, resultados, impactos y logros derivados de las acciones desarrolladas para el 
cumplimiento de los propósitos en la educación (Chaves-Manzano y Ordoñez-López, 2020). Este abordaje ha sido ampliamente difundido, y se ha promovido su aplicabilidad en distintos contextos, entre ellos, América Latina.

Al respecto, estudios como los de Díaz-Barriga (2020), Mosquera Albornoz (2018) y Rodríguez et al. (2018) coinciden en plantear que, si bien se reconoce el abordaje de la evaluación como un referente que desde la misma evolución de su concepción se ha orientado hacia el aporte en el desarrollo de procesos para la búsqueda de la calidad educativa, y el constante replanteamiento de las prácticas políticas, curriculares, pedagógicas, didácticas e incluso sociales, en el ámbito de la educación latinoamericana, resulta relevante analizar los avances, limitaciones y retos que se gestan en torno a la evaluación de la calidad educativa. Este es, precisamente, el propósito del presente artículo.

Así, atendiendo al objetivo propuesto, a continuación, se realiza un análisis teórico sobre el papel de la evaluación en la calidad educativa. Para ello, en un primer apartado se realiza una breve aproximación a la conceptualización de la evaluación, identificando sus puntos de encuentro con el abordaje de la calidad educativa. A partir de este referente se enfatiza particularmente en el papel de las prácticas evaluativas para el establecimiento de condiciones que posibiliten la calidad en la educación. Lo anterior, atendiendo a cómo se ha avanzado en la evaluación educativa y los principales aportes que esta le brinda al mejoramiento constante de los procesos que están inmersos en este ámbito. Finalmente, se señalan los principales avances, limitaciones y retos que desde el ámbito educativo han identificado distintas investigaciones en torno a la temática abordada. Todo ello con miras a generar un análisis que contribuya a seguir fortaleciendo los procesos educativos y el aprovechamiento de la evaluación en la búsqueda de mejoramiento constante que repercuta en una educación de alta calidad.

\section{Evaluación en el escenario educativo: Aproximaciones conceptuales}

La evaluación se constituye como un conjunto de procesos sistemáticos de indagación, que contribuyen deliberadamente a la generación de conocimientos mediante la valoración rigurosa de un fenómeno o de un conjunto de acciones que posibilitan el cumplimiento de propósitos establecidos en el ámbito educativo, y en el contexto donde este se enmarca (Jiménez Moreno, 2019). De esta manera, se alude a un análisis organizado y basado en criterios, normativas o relaciones, que contribuyen al monitoreo del quehacer en la educación y de las condiciones que permean el cumplimiento de los objetivos propuestos (Mosquera Albonoz, 2018; Ramírez González, 2011).

Al respecto, si bien en sus inicios se asumía la evaluación educativa como un mecanismo para el registro de resultados en los procesos de enseñanza-aprendizaje, su alcance ha trascendido para llegar a consolidarse como un referente inherente a todos los procesos educativos, y que da cuenta de condiciones iniciales (diagnóstico), avances y retrocesos 
http://doi.org/10.15359/ree.25-3.38

http://www.una.ac.cr/educare

educare@una.ac.cr

(proceso), así como resultados obtenidos e impactos generados en el marco del quehacer educativo. En este sentido, evaluar se refiere al proceso organizado que se realiza con el fin de establecer hallazgos tendientes a la comprensión de un fenómeno; sus características y los factores que están involucrados en este. Para ello, da lugar a procedimientos -muchas veces estandarizados y comparables- con miras a definir patrones de información desde los que se contribuya al análisis de personas, grupos, situaciones o fenómenos que se constituyen como objeto de evaluación (Borjas, 2014).

Así, en el ámbito educativo, la evaluación no solo hace parte del proceso de enseñanzaaprendizaje en el aula, sino que ha ido más allá para constituirse como un referente en la configuración misma del sistema educativo, y en el establecimiento de lineamientos para el ejercicio de la educación en los distintos contextos en los que ella tiene lugar. De hecho, cada vez se consolidan más estrategias para identificar con validez y precisión los cambios generados "en los conocimientos y capacidades de cada estudiante a lo largo del año lectivo- y/o de logro educativo-, entendido como la acumulación de conocimientos y capacidades a lo largo de toda la vida del estudiante" (Ravela, et. al., 2008, p. 5). Esto, con miras a establecer referencias en función del cumplimiento del currículo y de las exigencias de los organismos de control en las instituciones educativas. Sin embargo, además de estos referentes, la evaluación se ha constituido como un recurso de política pública y como un insumo para la calidad educativa.

En este punto resulta importante tener en cuenta que en la medida en que se alude a las evaluaciones como referentes para la toma de decisiones y la delimitación de lineamientos en el ámbito educativo, es importante tener claridad de su estructura, su propósito y el contexto donde estas se llevan a cabo. Solo de esta manera será posible aprovechar al máximo su potencial, reduciendo los riesgos a tergiversar información o generar interpretaciones erradas a partir de los resultados obtenidos en el ejercicio evaluativo. Se alude, por tanto, a la necesidad de comprender y abordar la evaluación desde su complejidad, atendiendo su utilidad, pero también sus limitaciones en el contexto educativo.

De hecho, la evaluación no se estructura ni se interpreta en el vacío; no se construye ni se comprende de espaldas a la realidad y al contexto en la que se enmarca, pues en torno a ella se teje una gran confluencia de factores que permean desde su estructuración y aplicación, hasta los resultados obtenidos a partir de ella. Muchos de estos factores no son observables, por lo que se hace alusión a un mecanismo valioso, pero complejo que debe abordarse con rigurosidad y organización para poder aprovecharlo al máximo, y dar cumplimiento a los objetivos que a través de este se pretenden alcanzar.

De allí que cuando se evalúa, no solo se mide un grupo de contenidos o ítems; también deben tenerse en cuenta los posibles elementos que median en el desempeño de esta acción para la educación. Siendo más precisos 
Para que la inversión en evaluación valga la pena, debe tener propósitos claros, una filosofía orientada a construir una visión de responsabilidad compartida en relación con la educación, un diseño técnico de calidad y adecuado a los propósitos, una orientación fuerte a apoyar a los docentes en su tarea y una voluntad política manifiesta de encarar acciones dirigidas a resolver los problemas y deficiencias que la evaluación ponga de manifiesto. (Ravela et al., 2008, p. 5)

Así, dada la complejidad inherente a la evaluación y el valor que tiene su uso en la configuración e implementación del quehacer educativo, esta debe asumirse como un insumo que va más allá de la presentación de datos que pueden percibirse muchas veces como medidas coercitivas para identificar errores y falencias presentes en el sistema educativo, o entre los actores que lo componen. No se trata, por tanto, de asumirlo como un recurso coercitivo que afecte el desempeño ni el quehacer educativo, pero tampoco se refiere a generar información para la atención de intereses particulares que no necesariamente respondan a las dinámicas y procesos propios del ámbito en el que se está llevando a cabo (Ahumada, 2005).

Las evaluaciones, si bien permiten identificar falencias, también dan cuenta de recursos y potencialidades que facilitan la creación de acciones efectivas para dar cumplimiento a los objetivos planteados en este escenario. En este punto, es importante tener en cuenta que las interpretaciones en el campo educativo no son lineales, pues los fenómenos sociales, los procesos de enseñanza-aprendizaje y el quehacer en la educación no se comportan bajo esa lógica. De allí que se debe ser cuidadoso con la interpretación y los usos de la evaluación.

Coherente con ello, resulta pertinente reiterar que la evaluación aporta, pero no se constituye como el único referente de la calidad en la educación. Asimismo, es relevante precisar que el potencial de la evaluación en la toma de decisiones y la generación de acciones tendientes a consolidar condiciones efectivas y útiles para el quehacer educativo se define en función del alcance mismo del ejercicio evaluativo. Lo anterior, teniendo en cuenta que, según los objetivos, metodología, actores involucrados, procesos de socialización y escenarios de aplicación de cada evaluación, se puede definirse cuál es la utilidad de los insumos que esta ofrece para la generación de reflexiones y el desarrollo de acciones de mejoramiento educativo (Jiménez Moreno, 2019; Mosquera Albornoz, 2018).

\section{El papel de la evaluación en la calidad educativa}

La calidad se refiere a un concepto sumamente complejo, tanto en su definición como en sus características e implicaciones. Al respecto, Bernal Suárez et al. (2015), y Cantón Mayo (2010) coinciden en definirlo como un conjunto de procesos orientados a posibilitar la consecución de objetivos, atendiendo a criterios que generan satisfacción a los sujetos destinatarios de un producto o servicio. Asimismo, se reconoce como el medio para promover la creación de valores agregados tanto a sus grupos objetivos como al sector donde se adscribe un producto o servicio determinado. 
http://doi.org/10.15359/ree.25-3.38

http://www.una.ac.cr/educare

educare@una.ac.cr

Este concepto se estructura inicialmente en el ámbito empresarial, constituyéndose como una forma de definir criterios relacionados con el control, para evitar posibles errores y consolidar prácticas enfocadas hacia la búsqueda de mejoramiento continuo. Coherente con lo anterior, la calidad se planteó inicialmente con el propósito de evaluar los resultados obtenidos a partir de la ejecución de actividades dirigidas a este fin, pero luego se reformuló para instaurarse como un referente inherente a todos los procesos que se desarrollaran en la empresa con miras a la consecución de sus objetivos organizacionales.

Este abordaje inicial de la calidad amplió su rango de acción a distintos escenarios que identificaron en este constructo la oportunidad de mejorar y potencializar sus procesos. Entre ellos, la educación fue uno de los escenarios en los que, durante la década de los 60, empezó a acuñarse el término. En sus orígenes, empezó a involucrarse en el ámbito educativo en función de la definición y medición de indicadores que dieran cuenta de la eficacia de las escuelas. En este sentido, la calidad se asumió en torno a la identificación de resultados en las instituciones educativas que dieran cuenta de las inversiones generadas en la época, así como la indagación en torno a los factores asociados a la efectividad de estos resultados con respecto a los recursos invertidos (Egido Gálvez, 2005).

Esta concepción de la calidad educativa ha venido replanteándose con el tiempo y, si bien aún no existe un criterio unificado con respecto a la misma, es posible plantear que este concepto alude al conjunto de condiciones socio económicas, políticas, institucionales y curriculares que facilitan la atención de las demandas y retos que se presentan en el marco del quehacer educativo. Así, la calidad educativa implica la formulación y consolidación de estrategias articuladas entre sí, con miras a la consecución de equidad, efectividad, oportunidad y acción contextualizada tanto en los lineamientos como en las prácticas educativas en las que esta se enmarca (Rodríguez Arocho, 2010; Seibold, 2000; Vásquez Olivera, 2015). Es, precisamente en este punto, donde confluyen la evaluación y la calidad educativa como dos referentes interdependientes para la construcción de condiciones orientadas a potencializar la educación como motor de transformación social.

\section{Evaluación para la calidad en educación: Avances, retos y limitaciones en América Latina}

En torno a la confluencia entre educación y calidad educativa, en el contexto latinoamericano se han estructurado avances a partir de las reformas educativas que han permeado la organización del sistema educativo, de las políticas y de las prácticas educativas, visibilizando, además, la necesidad de configurar procesos evaluativos que aporten al mejoramiento constantes de estos (Díaz-Barriga, 2020; Rivas y Sánchez, 2016; Rodríguez et al., 2018). A partir de ello, se han presentado múltiples avances, pero también retos y limitaciones que deben ser visibilizados para seguir potenciando el papel que tienen las evaluaciones en la identificación de hallazgos que posibiliten la creación de condiciones para generar 
calidad educativa. Esto, como un referente fundamental en la consolidación de procesos contextualizados y que respondan a las exigencias sociales desde el reconocimiento de los actores involucrados, y la interacción constante e intencional tanto con las instituciones como con los factores que están inmerso en el quehacer para la educación.

Con base en lo anterior, la evaluación en el ámbito educativo ha tenido avances importantes que van desde la concepción que se tiene de esta, hasta su estructuración y aprovechamiento en función de la calidad en sus procesos. Al respecto, en primer lugar, cabe resaltar que los sistemas de evaluación estandarizadas que han sido planteados para medir indicadores a gran escala, cada vez se hacen más precisos y tienen más en cuenta las dinámicas contextuales, los propósitos de aprendizaje y las funciones del sistema educativo para la configuración de las pruebas que son aplicadas. Aquí, si bien aún existen grandes limitaciones en estas, se reconoce la perspectiva de cambio que se ha asumido para el mejoramiento continuo de esos procesos estandarizados, que inicialmente se constituyeron como mediciones genéricas y comparables, sin tener en cuenta condiciones del entorno en el que se enmarcaba, para abordar una evaluación más holística y coherente con la complejidad que le es inherente a los procesos educativos (Jiménez Moreno, 2019).

De hecho, uno de los cambios que aporta a tal mejoramiento corresponde a la concepción que se ha empezado a consolidar en torno a la evaluación. Sobre este punto se resalta cómo se ha pasado de un abordaje evaluativo principalmente sumativo y ligado a los resultados (fundamentada en una perspectiva principalmente positivista), para empezar a abrir espacios en torno la idea de evaluación como un proceso reflexivo en sí mismo, que articula acciones para la formulación, ejecución e indagación de procesos, resultados e impactos de las prácticas educativas (generando una aproximación hacia perspectivas críticas, transformacionales y emancipadoras) (Ahumada, 2005; Borjas, 2014; Yarbrough et al., 2010).

Desde esta perspectiva, la evaluación con miras a la calidad educativa ha entrado en un proceso de transición donde más que estándares rígidos e inflexibles, cada vez se orienta más a la identificación de capacidades, potencialidades, condiciones favorables y oportunidades de mejora para fortalecer el quehacer educativo desde lo institucional y lo pedagógico. En esta misma línea, se sigue replanteando la concepción de lo evaluativo, pues, más allá de asumirse como un mecanismo para el planteamiento de indicadores que delimitan de manera unilateral "la calidad en la educación", ha dado lugar a un abordaje desde el que se asume como un insumo para la consolidación de dicha calidad, donde se tienen en cuenta múltiples factores involucrados en lo educativo, al tiempo que se constituye como un referente para la construcción de derroteros orientados a la transformación reflexiva de la praxis educativa (Díaz López y Ozuna Lever, 2016). 
http://doi.org/10.15359/ree.25-3.38

http://www.una.ac.cr/educare

educare@una.ac.cr

En este sentido, se consolida la concepción de evaluación para la calidad educativa desde una perspectiva de construcción continua y contextualizada, dirigida a la comprensión de las interacciones entre los distintos actores del sistema educativo, así como para el establecimiento de políticas públicas en la medida en que permite identificar posibles focos de atención para fortalecer la educación, al tiempo que posibilita el reconocimiento de recursos y oportunidades de mejora en su configuración e implementación. Todo ello a partir de la reflexión sistemática que puede generarse a partir de los hallazgos obtenidos con las pruebas, técnicas o instrumentos aplicados, para contribuir a la generación de acciones útiles para el mejoramiento de la calidad educativa (Ahumada, 2005; Calero y Choi, 2012; Yarbrough et al. 2010).

Otros de los avances identifica dos frente a este tema se refieren a la creciente tendencia de pasar de pruebas normativas dirigidas a organizar comparativamente al estudiantado según su desempeño, para empezar a consolidar instrumentos orientados a medir lo que saben y cuáles son las capacidades de los sujetos evaluados. Asimismo, ha empezado a darse lugar a criterios orientados a la definición de resultados que brinden información sobre cuáles deberían ser los resultados a obtener para que estos se consideren como satisfactorios. Complementariamente, se alude al reconocimiento y la confluencia de actores vinculados al escenario educativo (familia, docentes, comunidad, organizaciones empresariales, autoridades públicas) para fortalecer acciones de diseño en las evaluaciones, ser partícipes en las mismas evaluaciones, e incluso hacer parte de los procesos de divulgación y las actuaciones derivadas de estos (Vásquez Olivera, 2015; Zurita Rivera, 2020).

Para ello se han fortalecido las capacidades técnico - metodológicas que permiten la construcción de instrumentos y el procesamiento de datos obtenidos en estas pruebas, lo que permite identificar posibles insumos que aporten a la calidad, al tiempo que posibilita la estructuración de nuevas evaluaciones en función de las necesidades y requerimientos que surjan a partir en el marco de la búsqueda intencional, rigurosa y responsable del mejoramiento continuo. De la misma manera, vale la pena resaltar los esfuerzos para la transparencia en la difusión y el uso de los resultados obtenidos en las evaluaciones que se llevan a cabo, principalmente de forma masiva en la evaluación.

Cada vez se hace mayor énfasis en la relevancia de socializar los resultados obtenidos con una intención reflexiva y que aporta al cambio, así como de realizar procesos de acompañamiento desde lo gubernamental, lo institucional y lo pedagógico, para promover acciones y condiciones que favorezcan el mejoramiento a partir de la evaluación (Díaz-Barriga, 2020; Mosquera Albornoz, 2018). Se alude a un inicio importante en la trascendencia de privilegios a intereses particulares de los gobiernos de turno o de un conjunto de instituciones en especial, para dar lugar a la búsqueda de espacios para el aprovechamiento de los insumos que brinda el quehacer evaluativo en la calidad de la educación desde una perspectiva más amplia y contextual, pero que también atiende las dinámicas de una sociedad globalizada. 
http://doi.org/10.15359/ree.25-3.38

En esta misma línea se reconocen algunos avances en función de las evaluaciones internacionales y por competencias en el ámbito educativo. Estas prácticas han posibilitado la apertura frente a la reflexión y pautas para la acción educativa en función de las características y dinámicas de las sociedades actuales. Así, las evaluaciones educativas estandarizadas y masivas han aportado en la calidad educativa en la medida en que permiten tener en cuenta la situación de otros contextos desde una perspectiva de comparabilidad que puede repercutir en el mejoramiento del quehacer educativo a través de la valoración de modelos exitosos y la identificación tanto de factores protectores como de riesgo asociados a los resultados de las evaluaciones. Todo ello bajo una perspectiva crítica, analítica, rigurosa, dispuesta al aprendizaje y contextualizada de estos datos comparativos.

Sin embargo, en este punto cabe señalar que, si bien esta estrategia de comparabilidad es útil para visibilizar otros contextos y atender las demandas de organismos multilaterales, su implementación y uso se constituye como un medio y no como un fin en la educación, se reitera la necesidad de asumirla e interpretarla desde los contextos donde tiene lugar, y sin equipararla a una medida totalitaria y taxativa de calidad educativa (Calero y Choi, 2012; Niño Zafra y Gama Bermúdez, 2014).

Además de los avances definidos hasta este punto, en el marco de la evaluación como referente para la calidad educativa, se han identificado limitaciones que requieren atención con miras a seguir consolidando condiciones para su aprovechamiento óptimo. Una de las limitaciones apunta a que, en ocasiones, el Estado enfoca las evaluaciones, principalmente, hacia la asignación de recursos y la generación de incentivos en función de los resultados obtenidos, dejando en segundo plano el potencial formativo y de mejoramiento continuo que estas pueden generar (Rivas y Sánchez, 2016; Rodríguez et al., 2018). Asimismo, la lectura que se hace frecuentemente de los resultados en las evaluaciones suele centrarse en la asignación de responsables -principalmente la familia y la escuela-. Se limita, de esta manera, la perspectiva evaluativa, asumiéndola como un asunto que concierne a un grupo en particular de acuerdo con el grado de favorabilidad obtenido.

En este sentido, suele evidenciarse que en múltiples ocasiones existe poca claridad en el propósito de las evaluaciones, lo cual repercute en su diseño, su uso y su aprovechamiento. De igual manera incide en posibles errores al momento de tomar decisiones sobre "la utilización de censos o muestras, a los grados y disciplinas que serán evaluados, la periodicidad de las evaluaciones, el tipo de pruebas y escalas de reporte, entre otros" (Ravela et. al., 2008, p. 10).

Además de lo anterior, en la medida en que no se tienen claros los propósitos de una evaluación, se crean imprecisiones sobre las acciones que pueden generarse a partir de los resultados obtenidos con ellas. Este es uno de los factores que se constituye como un riesgo a la hora de utilizar el componente educativo como referente y punto de partida en la creación 
http://doi.org/10.15359/ree.25-3.38

http://www.una.ac.cr/educare

educare@una.ac.cr

de condiciones para procesos de enseñanza-aprendizaje que sean efectivos; que aporten a la calidad en la formación de su comunidad educativa, y a la calidad de la educación. Todo ello desde el reconocimiento de la complejidad que le es inherente al quehacer educativo, y a "la necesidad de invertir en la creación de capacidades como herramienta principal para la mejora de la enseñanza y el aprendizaje" (Ravela et. al., 2008, p. 15). Un ejemplo de ello se identifica cuando desde las instituciones educativas, e incluso desde sus respectivos organismos públicos de control, se insta a consolidar procesos de enseñanza aprendizaje dirigidos a la consecución de resultados favorables en una prueba estandarizada o a la ubicación en un ranking particular. Aquí se asume la evaluación como un fin y no como un medio para la calidad educativa, desconociendo sus propósitos y potencial aprovechamiento en el mejoramiento continuo de la educación (Cabra Torres, 2008).

Otras limitaciones que persisten en torno a la evaluación en educación se refieren a la poca discusión pública sobre los temas, propósitos, criterios y mecanismos de evaluación para mejorar los procesos educativos, así como a la identificación y abordaje de factores asociados a los resultados obtenidos en las evaluaciones de manera que se fortalezcan diseños de investigación que complementen y consoliden la comprensión, análisis y aprovechamiento de la evaluación en este escenario (Jiménez Moreno, 2019). Para ello se requiere continuar con la formación y participación de especialistas para diseñar e implementar este tipo de evaluaciones, al tiempo que resulta necesario dar continuidad tanto a los procesos como a los equipos técnicos que faciliten la generación de conocimientos y el aprovechamiento de los insumos que brindan las evaluaciones en el contexto educativo.

Estas dificultades y las exigencias que desde la educación se vienen configurando una serie de retos orientados al constante fortalecimiento de la evaluación, y a su mayor aprovechamiento con miras a la calidad educativa (Borjas, 2014; Martínez Rikzo, 2013; Perassi, 2008). Uno de los retos apunta a consolidar las evaluaciones que se necesitan en los sistemas educativos, atendiendo a las dinámicas, necesidades y exigencias del contexto donde estos se enmarcan, así como a los propósitos que se tienen con la práctica evaluativa. Se plantea, por tanto, la necesidad de seguir replanteando la idea de evaluación como una medición coercitiva, como un mecanismo para visibilizar resultados o para atender acríticamente a exigencias de organismos tanto nacionales como internacionales.

Coherente con ello, resulta imperante abordar la evaluación desde un enfoque amplio, cuyo aprovechamiento se oriente desde los procesos; no solo desde los resultados. Se habla por tanto de una evaluación que permita el diagnóstico, la comprensión de los procesos, el análisis de resultados y la identificación de impactos que tienen las prácticas educativas en la configuración que permean los procesos de enseñanza-aprendizaje. Además, es importante que los hallazgos identificados desde las evaluaciones sean visibilizados. La evaluación debe darse a conocer, divulgarse, compartirse, validarse y utilizarse en el escenario educativo que tiene lugar. 
Esto, teniendo en cuenta que evaluar no se limita a la recolección e interpretación de datos. La información que provee es sumamente valiosa en la medida en que se constituye como un insumo para la caracterización de la realidad y para aportar en la configuración tanto de políticas como de acciones orientadas al mejoramiento constante de la calidad educativa. Así, resulta imprescindible asumir la evaluación desde la disposición al aprendizaje en función de los recursos que esta brinda.

En este sentido cabe resaltar la importancia de estructurar la evaluación desde perspectivas participativas, orientadas hacia la transformación y el cambio; no solo de los procesos de enseñanza-aprendizaje, sino también de la sociedad en la que estos se desarrollan. Aquí es importante que el personal docente sea partícipe de los procesos evaluativos, no solo como un mecanismo para identificar si se han cumplido o no los objetivos establecidos desde los lineamientos institucionales sino desde una perspectiva de aprendizaje continuo donde se evalúa para mejorar. Este abordaje requiere la creación de una cultura evaluativa que debe forjarse desde la educación inicial de las mismas personas docentes, pero que también debe consolidarse entre los distintos miembros de la comunidad educativa y de los actores que confluyen en el sistema educativo. Para ello resulta fundamental consolidar referentes conceptuales y metodológicos que briden pertinencia, precisión y claridad a los procesos evaluativos. De esta manera se posibilita la selección rigurosa de los grupos objetivos, métodos adecuados, posibles usos y potenciales impactos que aporten, desde la evaluación, al fortalecimiento de la calidad educativa.

\section{Conclusiones}

La calidad educativa se constituye como un constructo complejo que da cuenta de la confluencia de factores asociados a las condiciones que posibilitan el ejercicio óptimo y autónomo de la educación con todos los procesos que le son inherentes. En este sentido, hablar de calidad en la educación implica hacer referencia a un constructo que se estructura y repercute en el desarrollo socio económico y cultural del contexto en el que tiene lugar. Así, uno de los mecanismos para identificar los referentes que hacen posible la calidad es la evaluación. Esto en la medida en que permite conocer potencialidades, oportunidades de mejora y condiciones para, a partir de allí, generar acciones dirigidas al mejoramiento y a la transformación educativa. Esta transformación implica, además de conocimientos y habilidades, la construcción de acciones reflexivas, críticas e intencionales que tomen como referente las condiciones del contexto para, a partir de ella, contribuir al mejoramiento constante desde el ámbito individual, familiar, comunitario, escolar y social.

De allí que resulte fundamental reconocer la importancia de la evaluación en el ámbito educativo desde una perspectiva crítica y rigurosa, sin que esto se traduzca en la concepción de la evaluación como el único referente para reconocer el estado y condiciones educativas 
http://doi.org/10.15359/ree.25-3.38

http://www.una.ac.cr/educare

educare@una.ac.cr

en un momento y contexto determinados. Lo anterior, teniendo en cuenta que en la práctica evaluativa confluyen múltiples factores, pues se alude a un ejercicio expuesto en un contexto complejo, cambiante y donde deben tenerse en cuenta distintos elementos que no siempre son contemplados en las evaluaciones realizadas. Se requiere, por tanto, reconocer los avances, limitaciones y retos que tiene la evaluación en el ámbito educativo, atendiendo a que esto se constituye como un referente importante en la clarificación en torno a la conceptualización, estructuración, delimitación de alcances y posibles usos que esta pueda tener para el mejoramiento educativo.

\section{Referencias}

Ahumada, P. (2005). La evaluación auténtica: Un sistema para la obtención de evidencias y vivencias de los aprendizajes. Perspectiva educacional, formación de profesores, (45), 11-24. https://www.redalyc.org/pdf/3333/333329100002.pdf

Bernal Suárez, D., Martínez Pineda, M. L., Parra Pineda, A. Y., Jiménez Hurtado, J. L. (2015). Investigación documental sobre calidad de la educación en instituciones educativas del contexto iberoamericano. Revista Entramados-Educación y Sociedad, 2(2), 107-124. https:// dialnet.unirioja.es/servlet/articulo?codigo $=5236201$

Borjas, M. (2014). La evaluación del aprendizaje como compromiso: Una visión desde la pedagogía crítica. Rastros Rostros, 16(30), 35-45. https://doi.org/10.16925/ ra.v16i30.816

Cabra Torres, F. (2008). La evaluación y el enfoque de competencias: Tensiones, limitaciones y oportunidades para la innovación docente en la universidad. Revista Escuela de Administración de Negocios, (63), 91-106. https://doi.org/10.21158/01208160.n63.2008.445

Calero, J., y Choi, A. (2012). La evaluación como instrumento de política educativa. Presupuesto y gasto público. (67), 29-41. http://www.ub.edu/histodidactica/images/documentos/pdf/ GASTO\%20PUBLICO-67-02\%20CALERO.pdf

Cantón Mayo, I. (2010). Introducción a los procesos de calidad. Revista Iberoamericana sobre Calidad, Eficacia y Cambio en Educación, 8(5), 3-18. https://revistas.uam.es/index.php/ reice/article/view/4723/5157

Chaves-Manzano, H. R., Ordoñez-López, I. del P. (2020). Cavilaciones sobre la evaluación y la calidad educativa en Colombia. Revista Arbitrada Interdisciplinaria KOINONIA, 5(9), 66-85. http://dx.doi.org/10.35381/r.k.v5i9.267

Díaz-Barriga, Á. (2020). Andares curriculares en América Latina. Revista Enfoques Educacionales, 17(2), 1-14. http://dx.doi.org/10.5354/0717-3229.2020.60634 
Díaz López, K. M. y Osuna Lever, C. (2016). Las evaluaciones estandarizadas del aprendizaje y la mejora de la calidad educativa. Temas de educación, 22(1), 131-146. https://revistas. userena.cl/index.php/teduacion/article/view/741

Egido Gálvez, I. (2005). Reflexiones en torno a la evaluación de la calidad educativa. Tendencias Pedagógicas, (10), 17-28. https://dialnet.unirioja.es/servlet/articulo?codigo=1407961

Jiménez Moreno, J. A. (2019). Aproximaciones epistemológicas de la evaluación educativa: Entre el deber ser y lo relativo. Foro de Educación, 17(27), 185-202. https://doi.org/10.14516/ fde.636

Martínez Rizo, F. (2013). El futuro de la evaluación educativa. Sinéctica, (40), 1-11. http://www. scielo.org.mx/scielo.php?script=sci arttext\&pid=S1665-109X2013000100006

Mosquera Albornoz, D. R. (2018). Análisis sobre la evaluación de la calidad educativa en América Latina. Caso Colombia. Revista Iberoamericana de Evaluación Educativa, 11(1), 43-55. https://doi.org/10.15366/riee2018.11.1.003

Niño Zafra, L. S. y Gama Bermúdez, A. (2014). Las políticas educativas de competencias en la globalización: Demandas y desafíos para el currículo y la evaluación. Itinerario Educativo, 28(64), 37-64. https://doi.org/10.21500/01212753.1420

Perassi, Z. (2008). La evaluación en educación: Un campo de controversias. Ediciones LAE. https:// www.yumpu.com/es/document/view/14216654/la-evaluacion-en-educacion-uncampo-de-controversias

Ramírez González, A. (2011). El rigor metodológico en la evaluación curricular. Revista Electrónica Educare, 15(2), 33-39. https://doi.org/10.15359/ree.15-2.3

Ravela, P., Arregui, P., Valverde, G., Wolfe, R., Ferrer, G., Martínez Rizo, F., Aylwin, M. y Wolff, L. (2008). Las evaluaciones educativas que América Latina necesita. (Documentos N. 40). PREAL. http://archive.thedialogue.org/PublicationFiles/PREAL\%2040-Spanish.pdf

Rivas, A. y Sánchez, B. (2016). Políticas y resultados educativos en América Latina: Un mapa comparado de siete países (2000-2015). Revista electrónica de Investigación y Evaluación Educativa, 22(1), 1-30. https://doi.org/10.7203/relieve.22.1.8245

Rodríguez Arocho, W. (2010). El concepto de calidad educativa: Una mirada crítica desde el enfoque histórico cultural. Actualidades investigativas en Educación, 10(1), 1-28. https:// doi.org/10.15517/AIE.V10l1.10088

Rodríguez, L. R., Vior, S. E., Más Rocha, S. M. (2018). Las políticas de evaluación de la calidad educativa en Argentina (2016-2018). Educação \& Realidade, 43(4), 1405-1428. http://dx.doi. org/10.1590/2175-623684907 
http://doi.org/10.15359/ree.25-3.38

http://www.una.ac.cr/educare

educare@una.ac.cr

Seibold, J. R. (2000). La calidad integral en educación. Reflexiones sobre un nuevo concepto de calidad educativa que integre valores y equidad educativa. Revista Iberoamericana de Educación, (23), 215-231. https://rieoei.org/historico/documentos/rie23a07.htm

Vásquez Olivera, M. G. (2015). La calidad de la educación: Reformas educativas y control social en América Latina. Latinoamérica. Revista de Estudios Latinoamericanos, (60), 93-124. https:// doi.org/10.1016/j.larev.2014.10.001

Yarbrough, D. B., Shulha, L. M., Hopson, R. K. y Caruthers, F. A. (2010). The program evaluation standards ( $3 .{ }^{\mathrm{a}}$ ed.). Sage.

Zurita Rivera, U. (2020). La participación social en la reforma educativa en México 2012-2018. Actualidades Investigativas en Educación, 20(3), 1-28. https://dx.doi.org/10.15517/aie. v2013.43673 